\title{
EDITORIAL
}

\section{Celebrating 10 years since launch}

t has been 10 years since the launch of Nature Reviews

Clinical Oncology in November 2004, formerly branded

Nature Clinical Practice Oncology. The journal's founding Editor-in-Chief, Vincent T. DeVita Jr, wrote an opening editorial, which stated "We live in the most exciting time in the history of cancer medicine. The molecular revolution has reached the bedside." If 2004 was exciting, we can only gasp at the advances that have occurred in clinical oncology over the subsequent decade. The premise behind Nature Publishing Group launching clinical review journals in eight therapy areas was simple: we wanted to serve our clinicians in a way no other journal was achieving at that time.

One of the key goals of the journal was to act as a filter to identify and critically appraise the best research articles across the vast spectrum of translational and clinical publications, to ease the workload of busy physicians who grapple with keeping abreast of the literature. We introduced Practice Points (now renamed News \& Views) - a unique feature of the journal that allows independent experts to critically discuss influential research papers-to help distill and interpret the vast amount of clinical data. Nature Reviews Clinical Oncology has undergone some notable changes, but the ultimate aims and scope of the journal remain unaltered and unchallenged.

I remember back in 2004 when we were first selecting which articles to commission as Practice Points. There was massive excitement surrounding the two papers that had just been published in the New England Journal of Medicine showing that patients with NSCLC who had an EGFR

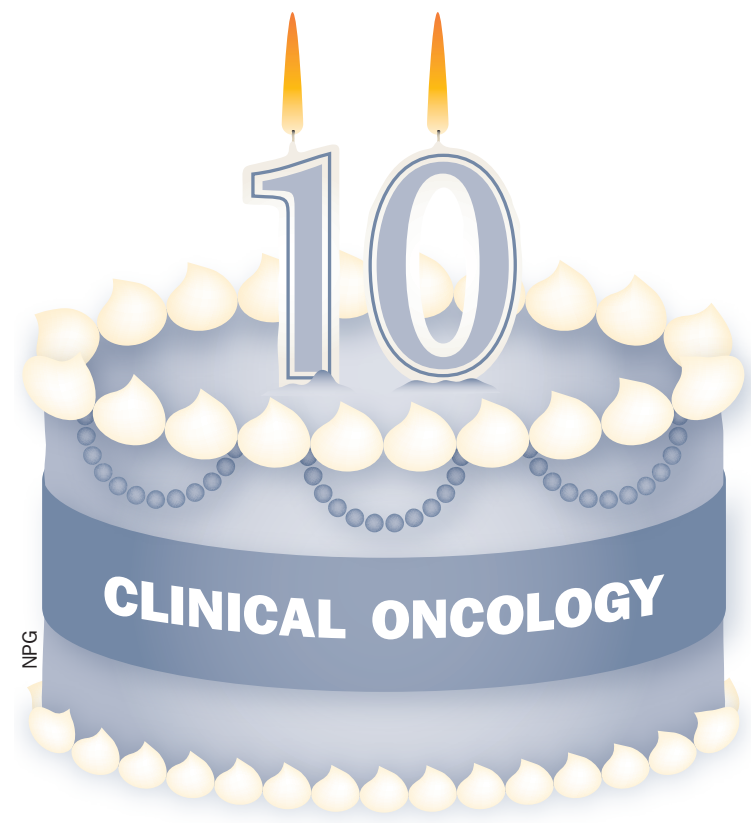

mutation were more responsive to targeted therapy with gefitinib. These findings represented one of the first examples of personalized medicine for solid tumours reaching the clinic - that the mutational status could predict therapeutic efficacy for a specific agent in a molecularly-defined subset of patients. These observations were groundbreaking and, along with Brian Druker's work on imatinib for treating CML, marked the epoch of the molecular targeted therapy era. Nevertheless, cancer fought back to circumvent our drug-based approaches to target such mutations, and acquired resistance is an area under active study. This is a candid reminder of how adaptable cancer is and what we are up against. But, we are learning and making tractable progress in the fight against the Emperor of all maladies. A notable example is the progress in our fundamental understanding of tumour heterogeneity and clonal evolution.

So much has happened in the past decade that we decided to commission six special Decade in Review articles by experts covering key areas of oncology: immunotherapy, haematological malignancies, genomics, targeted therapies and combination treatments, clinical-trial design and cancer-research funding, to chronicle the past 10 years of progress. We also invited four Advisory Board members associated with the journal since its launch-Vincent $\mathrm{T}$. DeVita Jr, Alexander M. M. Eggermont, Samuel Hellman and David J. Kerr-to discuss not only the outstanding success stories, but also to offer their insight into how best to tackle some of the frustrations and roadblocks we face in clinical cancer research today.

Over the past decade, I think we have achieved great things as a community, but the complexity of our infrastructure, societal challenges, spiralling costs and outdated clinical-trial paradigms that have not kept pace with scientific progress now threaten to be the biggest hurdles in combating cancer, rather than the intricacies of the disease itself. In addition, the number one priority in the next decade will be to understand better the mechanisms of acquired (and intrinsic) resistance as well as gaining insight from both the similarities and differences between normal cells, primary tumours and their metastases. I suspect that our progress in understanding cancer biology and the tumour and immune microenvironments will continue to throw up just as many questions as it provides answers.

During this past decade, it has been a privilege to have journeyed on this rollercoaster of discovery in clinical oncology, where I have learnt so much, especially from Vincent DeVita. I look forward to witnessing an equally exciting decade from November 2014.

doi:10.1038/nrclinonc.2014.164
4 ...but the ultimate aims and scope of the journal remain unaltered...77
Lisa Hutchinson is the Chief Editor of Nature Reviews Clinical Oncology.

Competing interests The author declares no competing interests. 